\title{
Biometria testicular e características morfológicas dos espermatozoides de cachorros-do-mato Cerdocyon thous Linnaeus, 1766 (Carnivora, Canidae)*
}

\section{Testicular biometry and morphological characteristics of crab-eating fox sperm Cerdocyon thous Linnaeus, 1766 (Carnivora, Canidae)}

\author{
Teresinha Inês de Assumpção, ${ }^{* *}$ André Luiz Quagliatto Santos, ${ }^{* *}$ Evandro Alves Canelo**
}

\begin{abstract}
Resumo
A pesquisa teve por objetivos avaliar a biometria testicular, coletar o sêmen e analisar as características morfológicas dos espermatozoides de cachorros-do-mato Cerdocyon thous Linnaeus, 1766. Foram utilizados cinco animais, maiores de três anos de idade e com peso superior a $6 \mathrm{~kg}$. Os animais foram anestesiados com xilazina $2 \%$, cloridrato de ketamina $10 \%$ e atropina $1 \%$. Foi determinado o volume testicular (testículos medidos com paquímetro). O sêmen foi coletado pelo método de eletroejaculação e a morfologia espermática analisada pelo método de preparação em câmara úmida, sendo realizado também o teste supravital (corante eosina/ nigrosina). O volume médio dos testículos foi $2,96 \pm 0,56 \mathrm{~cm}^{3}$. A análise da morfologia espermática teve média de $54,2 \%$ de defeitos totais e alta incidência de caudas dobradas e enroladas, gotas citoplasmáticas e defeitos no formato da cabeça. No teste supravital obteve-se $77,4 \pm 5,17 \%$ de células vivas. Conclui-se que os cachorros-do-mato (Cerdocyon thous) possuem volume testicular menor que outros canídeos selvagens; que a técnica de coleta de sêmen foi eficiente para a espécie e que o sêmen apresenta elevada patologia espermática, talvez devido à pequena variabilidade genética da população.
\end{abstract}

Palavras-chave: canídeo selvagem, reprodução, testículos.

\begin{abstract}
This research aimed to evaluate the testicular biometry, collect semen and analyze the morphological characteristics of the sperm of crab-eating fox Cerdocyon thous Linnaeus, 1766. Five animals older than three years-old and weighing more than $6 \mathrm{~kg}$ were used. The animals were anaesthetized with $2 \%$ of xylazine, $10 \%$ of ketamine hydrochloride, and $1 \%$ of atropine. The testicular volume was determined (testicles measured with caliper ruler). The semen was collected by the electroejaculation method, and the spermatozoa morphology was analyzed by the preparation in a humidified chamber method. The supravitally test was also performed (eosin /nigrosine pigment). The average volume of the testicles was 2,96 $\pm 0,56 \mathrm{~cm}^{3}$. Analysis of the morphology had a mean of $54.2 \%$ of total defects and high incidence of bent and rolled tails, cytoplasmic droplets and head shape defects. In supravitally tests were obtained $77.4 \pm 5.17 \%$ of alive cells. We conclude that the crab-eating foxes (Cerdocyon thous) have lower testicular volume than other wild canids, that the semen collection technique was efficient for the species, and that the semen has high sperm pathology, perhaps due to the low genetic variability of the population.
\end{abstract}

Keywords: wild canid, reproduction, testicles

\section{Introdução}

As ações humanas colocam em risco a biodiversidade do planeta e o desmatamento é o grande responsável pela destruição e perda de hábitat, o que leva a extinção das espécies. Entre estas espécies está o cachorro-do-mato (Cerdocyon thous) que é um animal onívoro, de porte médio, hábito preferencialmente noturno e habita quase todo o território nacional e tem ampla distribuição na América do Sul (Beisiegel et al., 2013).

O principal obstáculo para o sucesso da reprodução assistida em carnívoros selvagens são os conhecimentos ainda limitados sobre o comportamento e a fisiologia das espécies pertencentes a esse grupo. É muito importante possuir informações confiáveis sobre sazonalidade reprodutiva, puberdade, maturidade sexual e conhecer as características fisiológicas e morfológicas do sêmen dos machos (Micheletti et al., 2011).

Os parâmetros de biometria testicular são de grande importância no estabelecimento dos padrões reprodutivos e no desenvolvimento de protocolos para a reprodução assistida, pois há uma correlação direta entre o peso do testículo e a população espermática (Caldeira et al., 2010). Johnston et al. (2007) verificaram que cães selvagens africanos (Lycaon pictus)

*Recebido em 28 de março de 2017 e aceito em 24 de julho de 2017.

**Laboratório de Ensino e Pesquisa em Animais Silvestres, Faculdade de Medicina Veterinária, Universidade Federal de Uberlândia, Uberlândia, MG, Brasil. Autor para correspondência: teassumpcao@ufu.br 
têm o escroto não pendular e Souza et al. (2011) avaliaram a consistência do testículo de cachorros-do-mato em rígido, flácido ou normal (elásticos).

A coleta de sêmen em carnívoros silvestres é realizada por eletroejaculação, sob um protocolo anestésico, devido aos riscos ao animal e a equipe que o assiste e a dificuldade de manejo, sendo que a principal dificuldade encontrada para a realização deste método é a contaminação do sêmen por urina (Souza et al., 2011). Alguns fármacos utilizados na contensão podem interferir na qualidade seminal devido à contaminação do sêmen com a urina, pois promovem o relaxamento da região do colo da bexiga, e devido a proximidade da saída do ducto deferente, o sêmen tende a ir para a bexiga, causando a morte dos espermatozoides (Howard, 1993; Paz, 2013). Assim, é recomendada a drenagem da urina antes da coleta para minimizar o problema ou a cateterização da uretra na tentativa de coletar o sêmen sem contaminação com urina, o que tem sido utilizada com sucesso em felinos selvagens (Paz, 2013; Araújo, 2016).

$\mathrm{Na}$ avaliação da qualidade seminal verificam-se as características físicas e morfológicas do sêmen, sendo que as alterações na morfologia espermática são classificadas em anormalidades de cabeça, peça intermediária e de cauda (CBRA, 2013). Além disto, pode-se realizar coloração supravital, conhecida como coloração de vivos e mortos, cuja ação depende da integridade da membrana plasmática da cabeça do espermatozoide (Pugliesi, 2012).

Diante dos poucos conhecimentos da fisiologia reprodutiva dos cachorros-do-mato (Cerdocyon thous), os objetivos desta pesquisa foram avaliar a biometria testicular, coletar o sêmen e analisar as características morfológicas dos espermatozoides destes carnívoros selvagens.

\section{Material e métodos}

Foram utilizados seis cachorros-do-mato Cerdocyon thous Linnaeus, 1766 com massa corporal entre 5,4 e 7,4 kg, idade superior a três anos, em boas condições de saúde e sem nenhum tipo de lesão aparente. Os animais estavam alojados no Laboratório de Ensino e Pesquisa em Animais Silvestres da Universidade Federal de Uberlândia, Uberlândia/MG, oriundos de vida livre e encontrava-se em cativeiro após terem sido resgatados. Os mesmos eram mantidos em gaiolas individuais com alimentação apropriada para a espécie e água de boa qualidade.

Após jejum de 12 horas, os animais tiveram suas massas corporais medidas e foram contidos quimicamente sendo submetidos ao seguinte protocolo anestésico: xilazina $2 \%(1,13$ $\mathrm{mg} / \mathrm{kg})$, cloridrato de ketamina $10 \%(11,33 \mathrm{mg} / \mathrm{kg})$ e atropina $1 \%$ $(0,05 \mathrm{mg} / \mathrm{kg})$ por via intramuscular. O procedimento foi autorizado pelo comitê de ética em pesquisa envolvendo experimentação animal da Universidade Paranaense, protocolo 27332/2015. Os animais foram acompanhados clinicamente quanto à frequência respiratória e cardíaca por um médico veterinário especializado em animais silvestres durante todo o processo de coleta do sêmen e na recuperação pós-anestésica.

As avaliações dos animais e coleta do sêmen foram realizadas no mês de outubro (primavera). Os testículos foram avaliados quanto à consistência como rígido, flácido ou elástico. A biometria testicular foi realizada com auxílio de paquímetro digital $\left(\right.$ Pantec $^{\circledR}$ ) com exatidão de $0,03 \mathrm{~mm}$, para determinação do volume testicular, medindo-se o comprimento (C) e a largura (L), a fim de calcular o volume individual e total (V) do mesmo, utilizando-se a fórmula: $V=0,524 \times C \times L^{2}$ (Wildt, 1996; Johnston et al., 2007).

A coleta de sêmen foi realizada pelo método de eletroejaculação (figura 1), utilizando um eletroejaculador portátil (Autojac Neovet ${ }^{\circledR}$ - Brasil), acoplado a uma probe $(18 \times 2 \mathrm{~cm})$ que foi introduzida no reto do animal por aproximadamente $10 \mathrm{~cm}$. $O$ pênis do animal foi exposto manualmente para facilitar o recolhimento do sêmen. A série de estímulos seguiu o protocolo internacional utilizado para carnívoros selvagens, com 80 estímulos elétricos divididos em 3 séries: 30 (série 1:10 estimulações 2, 3 e 4 V), 30 (série 2:10 estimulações 3, 4 e 5 V) e 20 (série 3:10 estimulações 4 e $5 \mathrm{~V}$ ). Cada ciclo dos estímulos vai de aproximadamente 1 segundo da voltagem 0 até a desejada, permanecendo 2 a 3 segundos na voltagem desejada e retornando diretamente para a voltagem 0 por 3 segundos, com descanso de 3 minutos entre as séries (Howard, 1993).

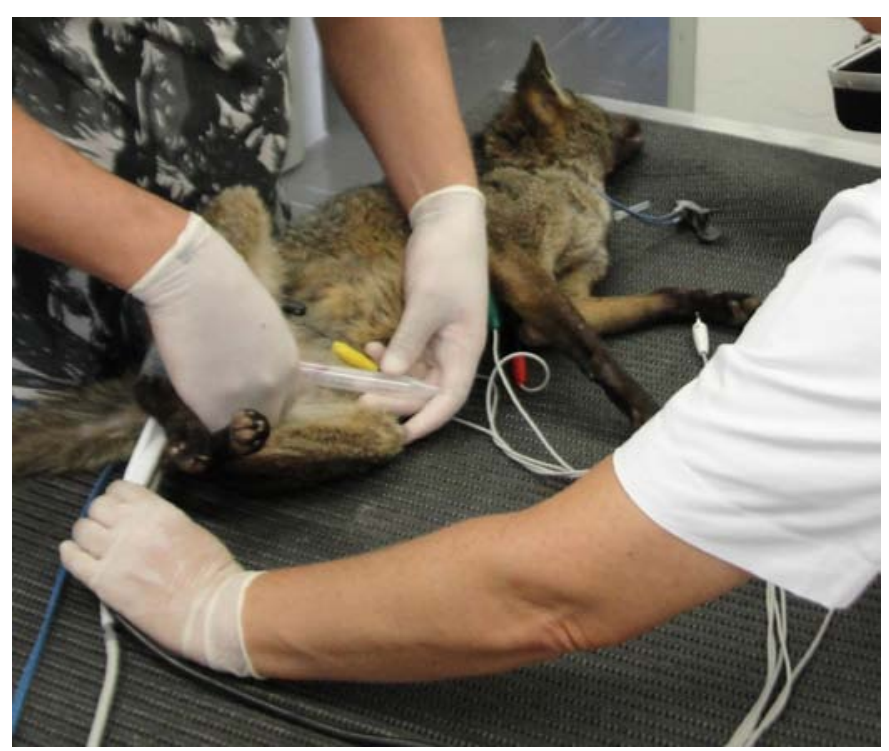

Figura 1: Fotografia de exemplar de Cedocyon thous. Procedimentos de coleta de sêmen por eletroejaculação.

Antes de proceder à coleta do sêmen foi utilizada uma sonda uretral $n^{\circ} 4$ estéril e lubrificado com gel, o qual foi inserido na uretra do cão e levado até a bexiga urinária para remoção da urina, utilizando uma seringa de $50 \mathrm{~mL}$ (Platz et al., 2001). Injetou-se na bexiga urinária do animal, com o uso da sonda uretral, $20 \mathrm{ml}$ de solução de PBS (tampão fosfato salino) para lavagem da mesma, fazendo o escoamento deste líquido em seguida.

Após os estímulos elétricos, o sêmen foi recolhido, tanto diretamente da uretra, quanto por aspiração da bexiga urinária (através da sonda uretral $n^{\circ} 4$ ), a fim de recuperar as células que tiveram seu fluxo retrógrado. Todo o material utilizado nas coletas (lâminas, tubos, etc) estavam aquecidos a $37^{\circ} \mathrm{C}$ para evitar choque térmico.

As amostras de sêmen foram analisadas morfologicamente de acordo com as normas do Colégio Brasileiro de Reprodução Animal (CBRA, 2013), utilizando o método de preparação em 
câmara úmida, sob microscopia óptica de contraste de fase, contando um total de 200 células em cada lâmina e analisandose os defeitos apresentados pelos espermatozoides em sua cabeça, peça intermediária e cauda, verificando a porcentagem de anormalidades (defeitos maiores e menores).

O teste supravital foi realizado utilizando um corante preparado com eosina $Y(3,3 g)$, nigrosina $(20 \mathrm{~g})$, citrato de sódio $(1,5 \mathrm{~g})$ e água destilada $(300 \mathrm{~mL})$. O esfregaço de sêmen foi feito misturando uma gota de sêmen e uma de corante, analisado em microscopia óptica, verificando o percentual de células vivas e mortas (CBRA, 2013).

\section{Resultados e discussão}

Os testículos de todos os animais apresentaram-se simétricos com consistência elástica, não havendo indícios de inflamações, degenerações ou outras alterações testiculares que pudessem alterar a qualidade do sêmen. A tabela 1 mostra as medidas médias do comprimento, largura e volumes dos testículos do cachorro-do-mato (Cerdocyon thous).

Tabela 1: Medidas testiculares $(\mathrm{cm})$ e volumes individual e total dos testículos $\left(\mathrm{cm}^{3}\right)$ dos cachorros-do-mato (Cerdocyon thous)

\begin{tabular}{ccccc}
\hline Animal & Comprimento & Largura & $\begin{array}{c}\text { Volume } \\
\text { individual }\end{array}$ & $\begin{array}{c}\text { Volume } \\
\text { Total }\end{array}$ \\
\hline 1 & 2,3 & 1,3 & 1,97 & 3,94 \\
2 & 1,8 & 1,3 & 1,59 & 3,18 \\
3 & 2,0 & 1,2 & 1,50 & 3,00 \\
4 & 2,0 & 1,1 & 1,27 & 2,54 \\
5 & 1,9 & 1,2 & 1,43 & 2,86 \\
6 & 1,8 & 1,1 & 1,14 & 2,28 \\
Média \pm DP & $1,96 \pm 0,18$ & $1,20 \pm 0,08$ & $1,48 \pm 0,28$ & $2,96 \pm 0,56$ \\
\hline DP - desvio padrão & & &
\end{tabular}

Os volumes verificados nos testículos dos cachorros-do-mato foram baixos, o que era esperado, pois os animais eram de porte corporal pequeno e possuíam também testículos pequenos. 0 volume médio encontrado foi $2,96 \pm 0,56 \mathrm{~cm}^{3}$, valor próximo ao verificado por Souza et al. (2011), que obtiveram para a mesma espécie o volume testicular de $3,4 \pm 0,4 \mathrm{~cm}^{3}$ (média de 3 animais) que foram avaliados no mesmo período do ano que os desta pesquisa. Relatos de Johnston et al. (2007) em cães selvagens africanos (Lycaon pictus), mostram testículos com volume bem mais elevados, sendo em média de $5,5 \pm 0,6 \mathrm{~cm}^{3}$ (peso corporal de 27 a $32 \mathrm{~kg}$ ) na primavera e 15,1 $\pm 1,5 \mathrm{~cm}^{3}$ (peso corporal de 29,5 a $36 \mathrm{~kg}$ ) no verão. Segundo Asa (1999), Johnston et al. (2007) e Minter e DeLiberto (2008) ocorre um aumento do volume testicular na estação reprodutiva com aumento da produção de espermatozoides nos canídeos selvagens.

A coleta de sêmen pela técnica de eletroejaculação foi bem sucedida em cachorros-do-mato, onde foi possível obter amostras suficientes para avaliação morfológica dos espermatozoides.

Dos seis animais avaliados, apenas de um não se obteve células espermáticas, pois o mesmo produziu apenas plasma seminal. Não foi possível realizar avaliação de motilidade e vigor espermáticos devido à contaminação do sêmen por urina, pois foi observada ejaculação retrógrada nestes animais, o que ocorre tanto em canídeos quanto em felinos tanto domésticos como silvestres, como relatado também por Johnston et al. (2007), Silva et al. (2008), Minter e DeLiberto (2008), Souza et al. (2011) e Paula (2011).

A tabela 2 mostra as quantidades médias individuais das diversas patologias espermáticas observadas em cachorrosdo-mato. A figura 2 ilustra a porcentagem de defeitos maiores, menores e totais do sêmen dos cachorros-do-mato. A figura 3 mostra as patologias espermáticas mais prevalentes no sêmen de cachorros-do-mato.

Tabela 2: Alterações morfológicas dos espermatozoides de cachorro-do-mato (Cerdocyon thous)

\begin{tabular}{lc}
\hline Tipo de alteração & $\begin{array}{c}\text { Média } \pm \text { EP } \\
\text { (porcentagem) }\end{array}$ \\
\hline Acrossoma & $0,4 \pm 0,24$ \\
Gota Citoplasmática Proximal & $11 \pm 5,06$ \\
Subdesenvolvido & $0,2 \pm 0,19$ \\
Cabeça Isolada Patológica & $0,4 \pm 0,39$ \\
Cauda Fortemente Dobrada ou Enrolada & $16 \pm 6,81$ \\
Pequeno Anormal & $0,4 \pm 0,24$ \\
Contorno Anormal & $8,0 \pm 3,74$ \\
Cauda Enrolada & $3,0 \pm 0,99$ \\
Cauda Dobrada & $5,6 \pm 1,02$ \\
Gota Citoplasmática Distal & $2,6 \pm 1,24$ \\
Cabeça Isolada Normal & $6,6 \pm 4,34$ \\
\hline EP - erro padrão &
\end{tabular}

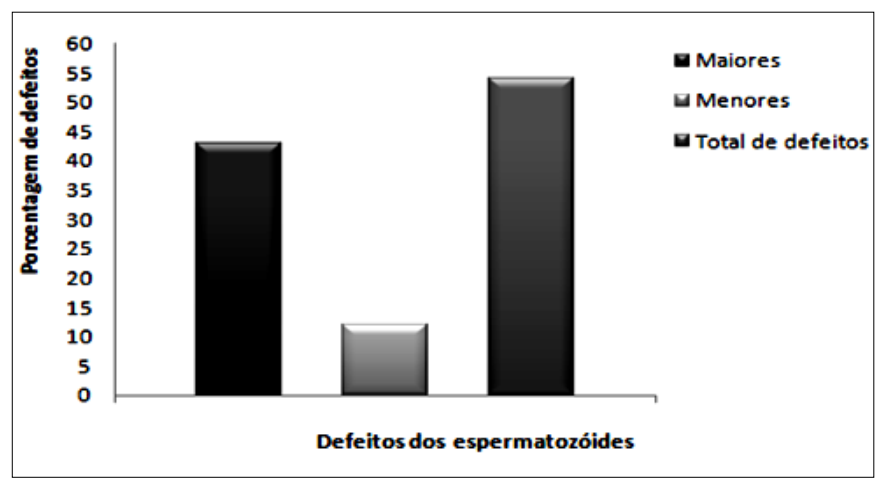

Figura 2: Percentual de defeitos maiores, menores e totais no sêmen dos cachorros-do-mato (Cerdocyon thous).

Não há padrões pré-determinados para qualidade seminal em cães selvagens, apenas para domésticos (CBRA, 2013). Verifica-se, neste trabalho, a média de $54,2 \%$ (21\% a $92 \%)$ de defeitos totais nos animais, com alta incidência de caudas dobradas e enroladas, gotas citoplasmáticas e no formato da cabeça (tabela 2), semelhante ao observado em cães selvagens africanos (Lycaon pictus) por Johnston et al. (2007), que verificaram em torno de $23,5 \%$ de patologias totais com alta incidência de gotas citoplasmáticas. Já Koeler et al. (1998), em lobo vermelho (Canis rufus) e Jalkanen (1993), em raposas prata (Vulpes vulpes) encontraram $35,0 \pm 11,8 \%$ e $45 \%$ de anormalidades do espermatozoide, respectivamente, com alta 


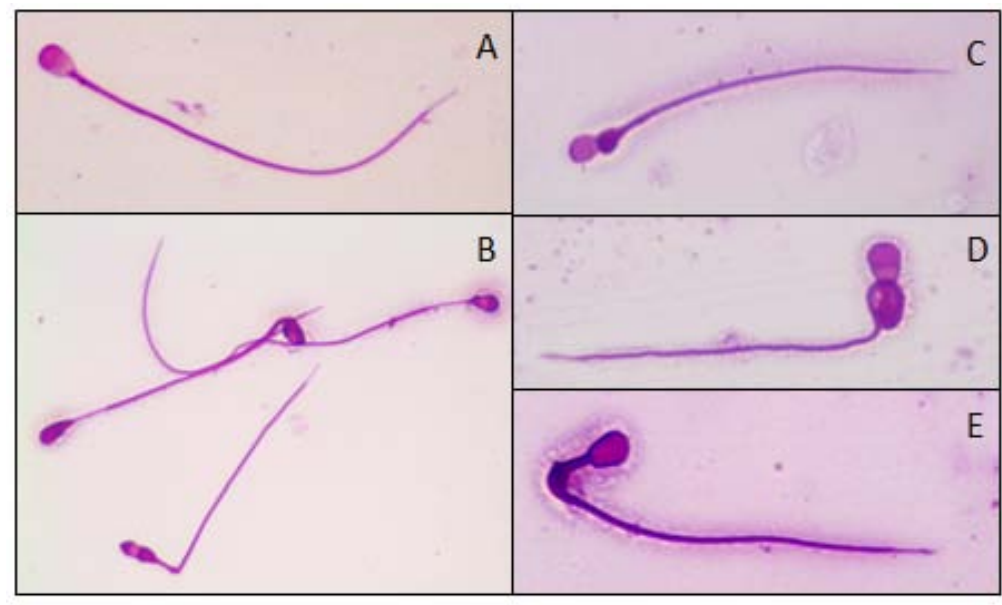

Figura 3: Fotomicrografias das alterações morfológicas dos espermatozoides de cachorros-do-mato (Cerdocyon thous). A) Morfologia do espermatozoide; B) contorno anormal da cabeça; C) gota citoplasmática proximal; D) e E) defeitos da cauda do espermatozoide. Coloração rosa bengala, aumento $1000 \mathrm{x}$.

incidência de defeitos de cauda, valores próximos ao observado em cachorros-do-mato (Cerdocyon thous). Por outro lado, em coiotes (Canis latrans), Minter e DeLiberto (2008) observaram $87,9 \pm 2,9 \%$ de células espermáticas normais, valor este muito maior que o encontrado neste estudo.

Pode-se inferir, talvez, que a elevada porcentagem de alterações morfológicas no sêmen se cachorro-do-mato (Cerdocyon thous) possa ser devido à pequena variabilidade da população desta espécie, devido ao baixo número de animais, e que sugere uma degradação na sua heterozigose (Wildt et al., 1986), o que foi observado também por Koeler et al. (1998) em lobos vermelhos, onde a restrição do pool genético e endogamia genética na pequena população remanescente explicaria a degradação observada na qualidade dos espermatozoides destes animais.

No teste supravital verificou-se grande número de células vivas, pois as células que possuem membranas íntegras não se coram, por não permitir a entrada do corante. Observou-se $77,4 \pm 5,17 \%$ de células vivas (não reativas ao corante) e $22,6 \pm 5,17 \%$ de células mortas (reativas à coloração).

\section{Referências}

ARAÚJO, G.R. Coleta farmacológica e criopreservação de sêmen de grandes felinos, mantidos em cativeiro e capturados em vida livre com o uso de armadilhas de laço: reprodução assistida em onças pintadas e onças pardas. 81p. Tese (doutorado), Universidade Federal de Viçosa, Viçosa, MG, 2016.

ASA, C.S. Dogs. In: KNOBIL, E., NEILL, J.D. (Eds.), Encyclopedia of Reproduction, Sydney: Academic Press, 1999, p. 902-909.

BEISIEGEL, B.M.; LEMOS, F.G.; AZEVEDO, F.C.; QUEIROLO, D.; JORGE, R.S.P. Avaliação do risco de extinção do Cachorrodo-mato Cerdocyon thous (Linnaeus, 1766) no Brasil.

Biodiversidade Brasileira, v.3, n.1, p.138-145, 2013.

CALDEIRA, B.C.; PAULA, T.A.R.; MATTA, S.L.P.; BALARINI, M.K.; CAMPOS, P.K.A. Morphometry of testis and seminiferous tubules of the adult crab-eating fox (Cerdocyon thous, Linnaeus, 1766). Revista Ceres, v.57, n.5, p.569-575, 2010.

CBRA. Manual para exame andrológico e avaliação de sêmen animal. 3ed. Belo Horizonte: CBRA, 2013.
A figura 4 mostra as células vivas e mortas do sêmen de cachorros-do-mato pelo teste supravital.

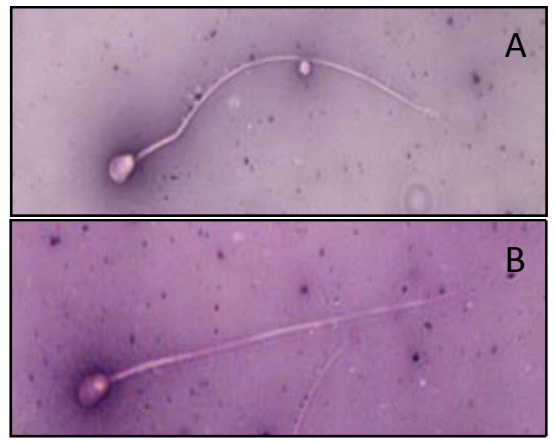

Figura 4: Fotomicrografias de espermatozoides não reativos $(A)$ e reativos $(B)$ ao teste supravital. Coloração eosina/nigrosina, aumento de $1000 \mathrm{x}$.

Em cachorros-do-mato (Cerdocyon thous) verificou-se elevada quantidade de espermatozoides com membranas plasmáticas íntegras, apesar da elevada patologia espermática detectada no sêmen destes animais. Resultado semelhante foi obtido por Johnston et al. (2007), que observaram $81,4 \pm 1,7 \%$ de células vivas (membranas intactas) no sêmen de cães selvagens africanos (Lycaon pictus), avaliadas com iodeto de propídio sob fluorescência.

Os conhecimentos limitados sobre o comportamento e a fisiologia reprodutiva dos cachorros-do-mato (Cerdocyon thous) são, ainda, o principal obstáculo para o sucesso da reprodução assistida nestes animais.

Conclui-se que os cachorros-do-mato (Cerdocyon thous) possuem volume testicular menor que outros canídeos selvagens; que a técnica de coleta de sêmen por eletroejaculação na espécie é eficiente em obter amostras para análise da morfologia espermática e que os espermatozoides apresentam elevada patologia, porém com grande quantidade de células de membranas íntegras.

HOWARD, J.G. Semen collection and analysis in carnivores. In: FOWLER, M.E. Zoo \& Wild Animal Medicine, Current Therapy, 3 ed., Philadelphia: W. B. Sauders, p.390-399, 1993.

JALKANEN, L. Sperm abnormalities in silver fox (Vulpes vulpes) semen selected for artificial insemination. Journal Reproduction Fertility, v.47, p. 287-290, 1993.

JOHNSTON, S.D.; WARD, D.; LEMON, J.; GUNN, I.; MACCALLUM, C.A.; KEELEY, T.; BLYDE, D. Studies of male reproduction in captive african wild dogs (Lycaon pictus). Animal Reproduction Science, v.100, p.338-355, 2007.

KOEHLER, J.K.; PLATZ, C.C.Jr.; WADDELL, W.; JONES M.H.; BEHRNS, S. Semen parameters and electron microscope observations of spermatozoa of the red wolf, Canis rufus. Journal of Reproduction and Fertility, v.114, p.95-101, 1998.

MICHELETTI, T.; CUBAS, Z.S.; MORAES, W.; OLIVEIRA, M.J.; KOZICKI, L.E.; WEISS, R.R.; MOREIRA, N. Reprodução assistida em felídeos selvagens - uma revisão. Revista Brasileira de Reprodução Animal, v.35, n.4, p.408-417, 2011. 
MINTER, L.J.; DELIBERTO, T.J. Seasonal variation in serum testosterone, testicular volume, and semen characteristics in the coyote (Canis latrans). Theriogenology, v.69, p. 946-952, 2008.

Paz, R.C.R. Reprodução de felinos domésticos e selvagens. Edufmt, 125 p. 2013.

PAULA, T.A.R. Reprodução de carnívoros silvestres. Revista Brasileira de Reprodução Animal, v.35, n.2, p. 103-132, 2011.

PLATZ, C.C., WADDELL, W., SMITH, R., JONES, M., BEHRNS, S., TEMPLETON, J. Semen collection in wild canidae using electroejaculation. In: LOSKUTOFF, N. M., CRICHTON, E. G. (Eds.), Proceedings of the First International Symposium on Assisted Reproductive Technology for the Conservation and Genetic Management of Wildlife. Henry Doorly Zoo, Omaha, USA p. 134-135, 2001.

PUGLIESI, G.; FURST, R.; CARVALHO, G.R. Efeito de diferentes tempos de equilíbrio na criopreservação de sêmen de garanhões. Revista Brasileira de Ciência Veterinária, v.19, p.172-177, 2012.

SILVA, T.F.P.; DIAS, C.G.A.; CARDOSO, J.F.S.; UCHOA, D.C.; BRAGA, A.C.P.; ACKERMANN, C.L.; PINHEIRO,

F.T.S.; BRILHANTE, D.F.M.; TAVERNEZI, L.; QUINTO, H.R.; SILVA, L.D.M. Comparação de quatro protocolos anestésicos para a coleta de sêmen por eletroejaculação em gatos domésticos. Ciência Animal, v.18, p.15-23, 2008.
SOUZA, N.P.; GUIMARÃES, L.D.A.; PAZ, R.C.R. Dosagem hormonal e avaliação testicular em cachorro-do-mato (Cerdocyun thous) utilizando diferentes protocolos anestésicos. Arquivo Brasileiro de Medicina Veterinária e Zootecnia, v.63, n.5, p.12241228, 2011.

WILDT, D.E. Male reproduction: assessment, management and control of fertility. In: KLEIMAN, D.G., ALLEN, M.E., THOMPSON, K.V., LUMPKIN, S. (Eds.), Wild Mammals in Captivity: Principles and Techniques. Chicago: The University of Chicago Press, $p$. 429-450, 1996.

WILDT, D.E.; SCHIEWE, M.C.; SCHMIDT, P.M.; GOODROWE, K.L.; HOWARD, J.G.; PHILLIPS, L.G.; O'BRIEN, S.J.; BUSH, M. Developing animal model systems for embryo technologies in rare and endangered wildlife. Theriogenology, v.25, n.1, p.33-51, 1986. 